\title{
Pattern Reconginationand Security
}

\author{
K.V Rajesh
}

\begin{abstract}
: there is a drastic change in the communication networks, majorly in the internet networks this has changed the entire life of a human being. In many of a business and organizations in common benefits, but in the same side there is the communication networks facing the major issues by the cyber-crimes. Which leads to the collapse entire the financial growth and result in the interruptions of a services. In protecting of a these networks in contradiction of a cyber-crimes is increasing interest in the cyber security communication. Basics of a the attacks pattern recognition by correlating and collecting cyber situational information precipitously through protocol levels and the parallel along the point to the point network paths are proposed in this paper. This leads to the decreases and analysis of a cyber-crimes from different corners of a world and improvement of a countermeasures.
\end{abstract}

\section{Keywords: cyber-crimes, cyber security, attacks pattern}

\section{INTRODUCTION}

Correspondence frameworks are nowadays seen as fundamental structures [1]. Unfortunately, the peril of a these frameworks tumbling on account of a cyber-attacks has moreover extended definitely. Resilience,is the limit of a framework to the keep up normal degrees of a movement despite various sorts of a challenges, the subject of a this paper, checking (D) DoS and various sorts of an advanced attacks, mis-structures [2], and operational over-burdens. Countries around the world are submitting their benefits for the fight to the come advanced challenges regardless they all agree that it is twisting up continuously inconvenient [3] as cyber law breakers are winding up progressively dealt with and present day. One instance ofsuch an attack is Ghost Net1 which has debased huge amounts of a PCs in various countries, which close to the $30 \%$ can be considered as political, high-regard key, or military targets, monetary. A couple of a research attempts have focused on the improvement of anadaptability disclosure and request methodology to the build cyber situational care reliant on this information.Moreover, sort out data has ended up being progressively open to the new gadgets and advances that give information about framework watching and applications direct. An immense section of an instruments to the screen structures and applications, in any case, perform evaluation on data from single sources, or datasets. Or then again potentially, for example using information from application layer firewalls to the catch attempted web application ambushes, we plan to the use arranged datasets at the same time, NetFlow looks for after for events over the data connection layer, to the give shocked computerized situation care. Which can be sorted out and associated with concentrate captivating confirmation about attack plans,

Revised Manuscript Received on December 12, 2019

K.V Rajesh, Department of CSE, Malla Reddy College Of Engineering For Women, Maisammaguda, Dhulapally, Kompally, Medchal (M), Secunderabad, Telangana 500100
These datasets are routinely available as logs, Models give a profitable system for speaking to and reusing learning. Their inspiration is to the pass on shown diagrams in a particular space. The use of a models has move out of a structure work and has been applied to the programming working by Gamma et al. [4]. Our adaptability essentials endorse that a spotlight should be made plans to the strategy for layered breaking points by get-together and interfacing advanced situational on a level plane along in the from of beginning to the end sort out way and information vertically across over show levels.

In pushing toward answers to the all the above referencing we may need to the diverse tremendous data from different datasets utilizing relationship of, timing, IP spaces, for instance and direct (e.g., under (D) DoS trap lead could be the degree of a degrees of a gatherings of a server ports to the mean number of an all assistance ports, streams each second per interface, and so forth.). This makes the supposition (to be kept up), that particularly coordinated and complex assaults are genuinely reflected in related models. Relationship is utilized to the depict the framework and unavoidable delayed consequences of a setting up huge association between different information things from various sources, routinely from changing and free sensors that screen structure and application occasions. Parts 3 and 4 clarify the proposed model and our point of view uninhibitedly.

This noteworthy information, is wanted to the include real risks dynamically or potentially in post-event evaluation for grasping and organizing as a rule adaptability controls for frameworks.

A model circumstance is laid out in Sect. 5. Portion 6 discussions about rapidly the future work ultimately wraps up the paper.

\section{LITERATURE REVIEW}

We would like to a development a profitable and productive structure that can pick computerized ambushes including unending related events. Trap vectors are typically everything considered dismissed on the Internet and their lifetime changes, which can make them hard to a see. In light of a nature, separating ambushes is an awkward task. We will practice unequivocal data mining and learning introduction checks, to the assistance us with discovering enrapturing catch plans. In the long run we are investigating explicit gathering strategies, for instance, Hierarchical, K-means and Graph based collecting [5-7], and looking colleague and sensibility all together with the achieve destinations for our proposed model. When in doubt, the system of an ambush assertion and requesting has been gotten some information about by using the assessment of an individual datasets, for example, Net Flow records, Server logs, and Web IDS logs, etc [8-10].

DOI:10.35940/ijeat.A1033.1291S619 
In any case, applying these structure systems to the single dataset isn't significant for seeing pervasive catch considers. In like manner, since ambushes advance after some time we see that applying those structures on a single dataset would not yield full scale affirmation about express attacks.

This organization includes:

(b)Unique ID and Name of a the attack configuration

(c) Describing information

(d) Attack methods and example

(e) Associated attack pattern.

The MITRE2 Corporation gives an uninhibitedly open stock of anambush models known as CAPEC Common Attack Pattern Enumeration.The list offers portrayal to the ambush structures close by broad framework and course of anaction logical arrangement. The ambush structure thought from CAPEC addresses a delineation of a normal attack strategies distracted from a great deal of a known authentic undertakings. CybOX is an organized language for encoding and giving high commitment information about computerized observables, paying little respect to the whether dynamic events. As a part of a CybOX Cyber Observable Expression, CAPEC is given which is a regulated model and is written in XML Extensible Markup Language.CybOX gives a commonplace structure and substance framework for tending to the cyber observables transversely finished. Among different use cases for by and large cyber situational care. In CAPEC, ambush models can be seen as dignified scholarly delineations of a huge level attacks with respect to the their traits, methods for abusing programming, for instance, structure vulnerabilities, weaknesses,. To the some degree, CAPEC attempts to the portray ambush models using a top-down approach, i.e., recording attacks and perceiving their key parts from the aggressor's perspective. In this paper we base on the get-together and association of an evidence from various datasets in order to the recognize instances of a related events that could explain an attack. Consequently, we consider the to the be approaches as enhancing each other, which could be in this manner insinuated as observables in CAPEC's attack plans, as in we will have the choice to the perceive and think ambush features, In [13], the maker has developed a botnet revelation structure subject to the gathering of a $\mathrm{C} \& \mathrm{C}$ correspondence and activities streams to the perceive closeness models and blend of a the two sorts of a models by cross-association. In this work isn't exactly equivalent to the own as we intend to the develop progressively expansive models that can be applied to a disclosure, request of the extent of a cyber-attacks rather than a particular technique that is engaged at a single sort of an ambush. Relationship has moreover transformed into the most noteworthy strategy for framework the board. In any case, at present, event relationship is generally used for framework the officials and we expect to the loosen up this to the various spaces, for instance, cyber situational care over different levels. Eventually, all these relationship systems are unequivocal to the single datasets and don't give complete comprehension by joining heterogeneous association across over various levels. (a) Associatedvulnerabilities and weakness

There are a couple of a other definitely comprehended endeavors that are used to the screen all unconstrained traffic facilitated to the dull subnets. Diminish nets are unused IP subnets, and various endeavors work darknets, for instance, the Team Cymru darknet [18] and the Internet Motion Sensor [19]. From different points of a view, vertically transversely over show levels and on a level plane along the all the way organize way. In these are regularly simply little bits of a general enigma, which are simply prepared to the give a deficient picture of a cyber works out.

\section{MODEL}

Standard models depict how to the adjust to the discretionary challenges $[9,10]$. In any case, we intend to the loosen up these models to the oversee especially refined attacks over the various levels in frameworks to the give hindrance controls against such attacks. In the present correspondence arrange circumstances, events and alarms begin from various self-governing sources [24].

Disclosure advances have made after some time, and sensibly irrefutable significance and broadness of an information is open for evaluation, for the most part refreshed with metadata and real information. Occasions of a such datasets include: trap events (honey net logs), make looks for after, and web crawler logs. It is without assistance for seeing ambushes over various data sources that we see the requirement for new research. This is in light of a the route that there is no framework to the give us markers for sufficiently doing fighting advanced attacks through checking out course of an action from various data sources, which controls the open entryways for a counter-measure development process. As such, interminably complete connection care needs to the development this far reaching information.

The critical level outline of a proposed model for ambush plan affirmation is given underneath :

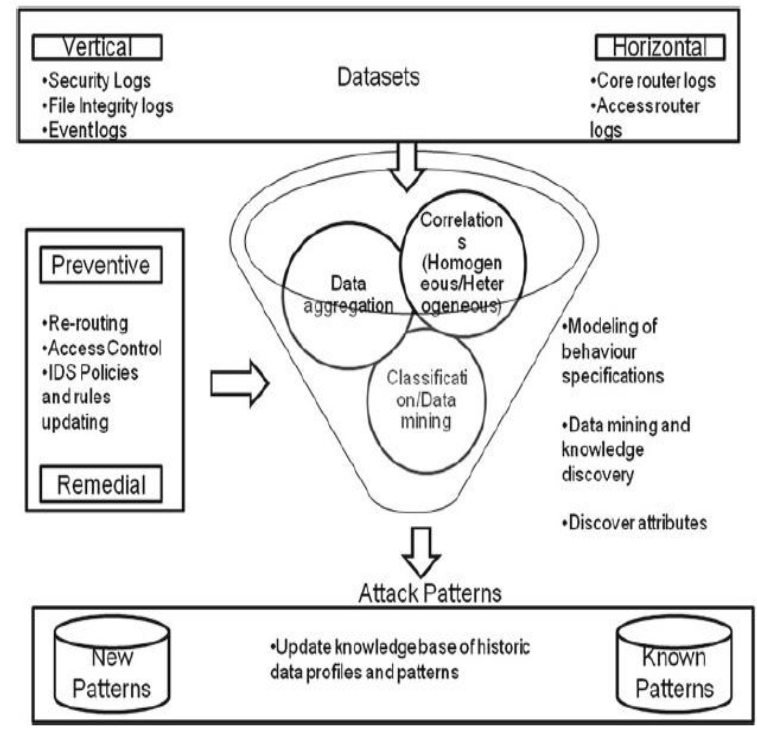

Fig. 1 High level strategy of a planned model for attack pattern appreciation. 


\section{APPROACH}

One of a the basic insights driving our philosophy is the attestation of a catch structures, which address a social gathering of a related events, with the vulnerability that, for a given attack, in any event two view centers will be open. Bewildering attempts have been made to the apply data mining methodologies to the issues related to the framework security, and these undertakings have fused the usage of a these procedures to the interface assessment, neural structures and other AI approaches for impedance perceiving affirmation. In any case, these undertakings rely upon moves up to an impedance zone system instead of finding affirmation or disclosure of another bits of a learning into secluding all out miracles of an ambush.

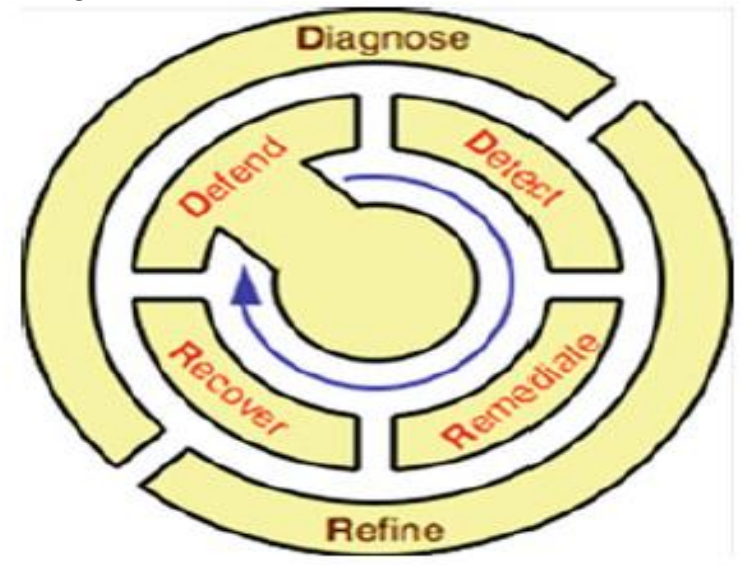

Fig. 2 Resilience strategy

Utilizing different datasets. What's more, just couple of a run of a the mill datamining procedure, for example, strategy calculations, partnership models have been applied to the foul system information in any case again it made courses of a action for improving the readied depiction to the overhaul the presentation of a the impedance exposure structure. Also, to the discover obscure snare plans we propose the use of a solo game-plan, as melancholy earlier getting some answers that may come to the fruition in perspective on blending check disconnected from various sources.

Quality is the farthest point of the system to the give up and keep great degree of a association even with different blames and difficulties to the standard activities [25]. Resume Net uses a general two-compose enormous level system adaptability methodology. The ResumeNet3project gives a significant strategy of a standards and arrangement rules for our model. The main stage incorporates the utilization of a careful (for example security attempts) to the shield the structure from obvious difficulties, for example, separating verification of anissues coherently, cyber-attacks and properly remediation, their assets before structure development is undermined, in end recuperation frameworks. The following stage on a very basic level joins improving help levels through the attestation and refinement of a tasks (see Fig. 2).

Energized by the essential parts in the model, this noteworthy level quality philosophy are the going with (see Fig. 3):

Stage 1: Discover and Compute: assurance of an attack features from dataset. concerning attacks is open. Thus, to the uncover structures

Stage 2: Selection and Extraction: grouping, which hopes to the discover meaning ful relations among models removed from the dataset

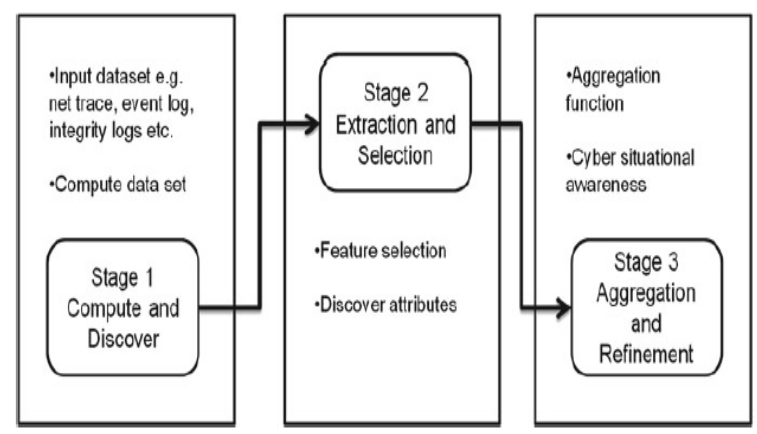

Fig. 3 Points of a the projected model

Stage 3: Refinement and Aggregation: The periods of a the model will be investigated using strong mixture, for instance the use of a data blend andupdating the learning baseof a remarkable data models and profiles models, for instance, the one in Sect. 5.

\section{Example of Attack}

Regularly, the objectives are obvious web servers. Aggressors can utilize a course of anaction of a frameworks to the bargain these structures, two or three attacks might be multi-sort out. The Distributed Denialof Service (DDoS) assault happens when different traded frameworks flood as far as possible or assets of the focused on structure, again regularly a web server. For instance, email spam and malware are utilized first to the deal with several structure focuses, by at that point, a (D) DoS trap might be started to the a predefined target. A central procedure to the envision such a relationship is to the envision a development of anoccasions as logs that are conveyed, each looking bits of the structure movement. Traffic highlights, time, for example, source IP address, and payload would then have the choice to the be utilized to the outline transport and structure. After standardization, these logs can be set more than one another so that by examining these layers one can perceive gigantic shared properties the degree that the snare point of a view.

\section{CONCLUSION}

In this paper, we propose a structure for cyber circumstance care in PC systems. We trust it is conceivable to the take a gander at various datasets with an aching for extending broad bits of a data into the OK collection and sorts of a the cyber issues pestering a structure. This is in light of a the way that the highlights of a these attacks make after some time, kind of a snare/misuse, source or target IP address, etc. The lifetime of the cyber-snare can change from days to the months, because oftheir propensity, attributing various wellsprings of a occasions to the a relative assault is an irksome errand. In like manner, the comfortable idea of anattacks would make it hard to the demonstrate their lead. A comparative assessment of a packaging and solicitation techniques for discovering security issues requires further research. 
In light of a powerlessness and irrelevant earlier information of a assault occasions, free assembling techniques will be our inside interest.

\section{REFERENCES}

1. Battista Biggio, Giorgio Fumera and Fabio Roli "Pattern Recognition Systems Under Attack: Design Issues And Research Challenges" International Journal of Pattern Recognition and Artificial Intelligence Vol. 28, No. 7 (2014) 1460002.

2. M. Antonakakis, R. Perdisci, Y. Nadji, N. Vasiloglou, S. Abu-Nimeh, W. Lee and D. Dagon, from throw-away tra $\pm c$ to bots: Detecting the rise of DGA-based malware, in 21st USENIX Security Symp. (USENIX, 2012), pp. 491-506.

3. I. Arce, The weakest link revisited, IEEE Security Privacy 1(2) (2003) 72-76.

4. A. Attar, R. M. Rad and R. E. Atani, A survey of image spamming and - ltering techniques, Artif. Intell. Rev. 40(1) (2013) 71-105.

5. M. Barreno, P. L. Bartlett, F. J. Chi, A. D. Joseph, B. Nelson, B. I. Rubinstein, U. Saini and J. D. Tygar, Open problems in the security of learning, Proc. 1st ACM Workshop on $\mathrm{Arti}^{-}$cialIntell. Sec., AISec'08 (ACM, 2008), pp. 19-26.

6. M. Barreno, B. Nelson, R. Sears, A. D. Joseph and J. D. Tygar, Can machine learning be secure? Proc. ACM Symp. Information, Computer and Comm. Sec., ASIACCS'06 (ACM, 2006), pp. 16-25.

7. A. Barth, B. I. Rubinstein, M. Sundararajan, J. C. Mitchell, D. Song and P. L. Bartlett, A learning-based approach to reactive security, IEEE Trans. Dependable Secure Comput. 9(4) (2012) 482-493.

8. B. Biggio, Z. Akhtar, G. Fumera, G. L. Marcialis and F. Roli, Security evaluation of biometric authentication systems under real spoo ng attacks, IET Biometrics 1(1) (2012) 11-24.

9. B. Biggio, I. Corona, G. Fumera, G. Giacinto and F. Roli, Bagging classi $^{-}$ers for ${ }^{-}$ghting poisoning attacks in adversarial environments, in 10th Int. Workshop on MCSs, eds. C. Sansone et al., LNCS, Vol. 6713 (Springer, 2011), pp. 350-359.

10. B. Biggio, I. Corona, D. Maiorca, B. Nelson, N. Šrndić, P. Laskov, G. Giacinto and F. Roli, Evasion attacks against machine learning at test time, in European Conf. Machine Learning and Principles and Practice Knowl. Discovery in Databases, Part III, eds. H. Blockeel et al., LNCS, Vol. 8190 (Springer, 2013), pp. 387-402.

11. B. Biggio, I. Corona, B. Nelson, B. Rubinstein, D. Maiorca, G. Fumera, G. Giacinto and F. Roli, Security evaluation of support vector machines in adversarial environments, in Support Vector Machines Applications, eds. Y. Ma and G. Guo (Springer International Publishing, 2014), pp. $105-153$.

12. B. Biggio, L. Didaci, G. Fumera and F. Roli, Poisoning attacks to compromise face templates, 6th IAPR Int. Conf. Biometrics (2013), pp. $1-7$.

13. B. Biggio, G. Fumera, I. Pillai and F. Roli, A survey and experimental evaluation of image spam ${ }^{-}$ltering techniques, Pattern Recogn. Lett. 32(10) (2011) 1436-1446.

14. B. Biggio, G. Fumera and F. Roli, Adversarial pattern classi ${ }^{-}$cation using multiple classi- ${ }^{-}$ers and randomisation, 12th Joint IAPR Int Workshop on Structural and Syntactic Pattern Rec., LNCS, Vol. 5342 (Springer-Verlag, 2008), pp. 500-509.

15. B. Biggio, G. Fumera and F. Roli, Evade hard multiple classi ${ }^{-}$er systems, in Supervised and Unsupervised Ensemble Methods and their Applications, eds. O. Okun and G. Valentini, Vol. 245, Studies in Computational Intell. (Springer, 2009), pp. 15-38. 